\section{KOMPASS}

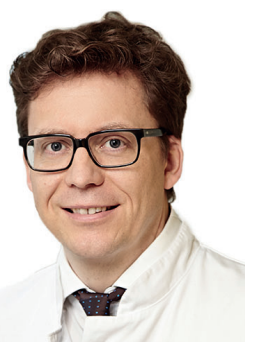

\section{Christian Schumann}

Klinik für Pneumologie, Thoraxonkologie, Schlaf- und Beatmungsmedizin, Klinikverbund Kempten-Oberallgäu, Kempten/Immenstadt, Deutschland

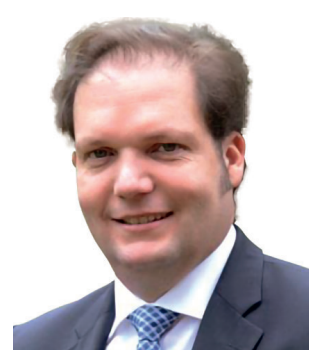

\section{Nicolas J. Dickgreber}

Klinik für Pneumologie, Thoraxonkologie und Beatmungsmedizin, Klinikum Rheine - Mathias-Spital/Klinikum Ibbenbüren, Ibbenbüren/Rheine, Deutschland

\title{
Lungenkrebs behandeln und begleiten - auf dem Weg zur chronischen Krankheit
}

Lungenkrebs ist mit rund 50000 Neuerkrankungen pro Jahr eine häufige Krebserkrankung in Deutschland. Zugleich ist die Sterblichkeit mit die höchste unter allen Krebserkrankungen. Ein Grund dafür ist, dass ein großer Anteil der Neuerkrankungen bereits zum Zeitpunkt der Erstdiagnose lokal fortgeschritten (Stadium III etwa 30\%) oder metastasiert (Stadium IV etwa 40\%) ist. Die bestimmende Frage ist daher, welche Entwicklungen das Potenzial zur Verbesserung der Prognose der an einem Lungenkarzinom Erkrankten haben, die wir in diesem Editorial stichpunktartig vorstellen möchten.

\section{Früherkennung}

Die Früherkennung wird in Zukunft eine wichtige Rolle dabei spielen, um mehr Patienten im sehr frühen Stadium I zu identifizieren und sie einer primären Operation zuzuführen. Nachdem eine Reihe von großen Studien in den USA (national lung trial, NLST) [1], aber auch in Europa (Niederlandisch-belgische LungenkrebsscreeningStudie, nederlands-Leuvens longkanker screenings onderzoek, NELSON) [2] eine deutliche Senkung der Mortalität (20-26\%) durch die Früherkennung zeigen konnten, sind in Deutschland bereits entscheidende Hürden (Gesetzesänderung, Einschätzung des Bundesamtes für Strahlenschutz, Beratungen der Fachgesellschaften, konkreter Zeitplan bis Ende 2020) genommen worden, um in naher Zukunft ein strukturiertes Lungenkrebsscreening anzubieten.
Neue neoadjuvante Konzepte

In den primär operablen Stadien II-IIIA gab es jahrzehntelang keine Innovationen. Bisher folgte nach der Operation die adjuvante Chemotherapie. Derzeit werden in klinischen Studien intensiv neoadjuvante Konzepte unter Einsatz von Immuntherapie und Chemotherapie geprüft. Am vielversprechendsten könnte eine Kombination aus Chemotherapie und Immuncheckpoint-Inhibitoren sein. Die Verbesserung der mPR (major pathologic response) bzw. CPR (complete pathologic response) sind dabei wichtige Kriterien und geben in bisherigen Studien dazu Hinweise auf eine erfolgreiche Induktion.

Immuntherapie im Stadium III Im lokal fortgeschrittenen Stadium IIIA-C, bei der primär eine Chemoradiotherapie indiziert ist, kann mit der konsolidierenden Immuntherapie bereits eine deutliche Verlängerung des Gesamtüberlebens erreicht werden (PACIFIC-Studie) [3]. Derzeit ist dafür Durvalumab bei Nachweis einer PD-L1-Expression von mindestens $1 \%$ zugelassen. Dabei ist die Immuntherapie für eine Dauer von 12 Monaten nach Beendigung der Strahlentherapie angezeigt. In einer Subgruppenanalyse der PACIFIC-Studie konnte vielmehr ein Vorteil für die Patienten gefunden werden, die eine enge zeitliche Kopplung der Immuntherapie an das Ende der Bestrahlung hatten (14 Tage). Die pulmonale Toxizität war dabei kein Problem.

\section{KARGER}

Fax +4976145207 14 information@karger.com www.karger.com (c) 2019 S. Karger GmbH, Freiburg
Prof. Dr. Christian Schumann

Klinik für Pneumologie, Thoraxonkologie, Schlaf- und Beatmungsmedizin

Klinikverbund Kempten-Oberallgäu $\mathrm{gGmbH}$

Im Stillen 3, 87509 Immenstadt, Deutschland

christian.schumann@kv-keoa.de 
Daher greifen aktuelle Studienkonzepte bereits den simultanen Einsatz von Immuncheckpoint-Inhibitoren im Rahmen der Chemoradiotherapie auf. Bisher lassen die Daten dazu eine nochmalige Verbesserung der Therapieergebnisse erwarten.

\section{Molekulare Testung und molekulares Tumorboard}

Im Stadium IV beim nicht kleinzelligen Lungenkarzinom (non-small cell lung carcinoma, NSCLC) kommt der molekularen Testung eine besondere Bedeutung zu, auch wenn diese in der Gesamtheit der direkt behandelbaren Mutationen (EGFR, ALK, ROS1, BRAF) nur 10-15\% ausmachen. Ein weiterer großer Teil an Alterationen wird im Rahmen von breiten Panel-Testungen (NGS (nextgeneration-sequencing)-Verfahren) identifiziert. Auch diese sind teils Therapien zugänglich, z.B. Off-Label- oder Compassionate-Use oder im Rahmen von klinischen Studien. Empfehlungen darüber geben molekulare Tumorboards.

Die daraus resultierende zielgerichtete Therapie kann gegebenenfalls mehrährige progressionsfreie Verläufe ermöglichen; das Gesamtüberleben ist klar länger als mit der bisherigen konventionellen Chemotherapie.

\section{Rebiopsie und Flüssigbiopsie}

Zudem ist im Fall von Treibermutationen eine besondere Bedeutung für die Rebiopsie zu unterstreichen. Der Nachweis von Resistenzmutationen, z.B. T790M, kann initial (de novo), aber auch im Verlauf bei Progress der Erkrankung eine Indikation für eine Therapieumstellung sein.

Neben der Testung im Gewebe ist auch dies per Flüssigbiopsie möglich. Spezielle
Testsysteme lassen je nach Analytik hochsensitive Bestimmungen aus dem Blut, aber auch aus Liquor oder Ergüssen zu. Auch im Falle einer Resistenz gegenüber ALK-TKI (anaplastic lymphoma kinase tyrosine kinase inhibitor) kann - auch wenn es derzeit noch keine Routine ist - eine Flüssigbiopsie mittels Variantenanalysen (RNA-basiert) darlegen, welche molekulare Resistenz vorliegt und welche Folgetherapie am besten geeignet sei.

Neue Ansätze beschreiben ein verbessertes Outcome bei Eintreten einer sogenannten Plasma-Clearance der zuvor beschriebenen Mutation, z.B. bei EGFR in der Flaura-Studie [4] (Vorteil). Kontrovers wird die Datenlage bei bTMB (blood based tumor mutational burden) diskutiert. Teils sprechen Daten diesen Biomarkern eine prädiktive Rolle zu (MYSTIC) [5], teils aber auch noch eine prognostische Rolle zu (KEYNOTE-189 und -407) $[6,7]$ oder mit Verweis auf die Gewebe-TMB-Testung eine ebenfalls prognostische Rolle (CheckMate227) [8]. Hier sind aber noch Aufgaben wie Harmonisierung der Testung (Umfang, Methodik, Analytik und Interpretation) anzugehen.

\section{Damit ist eine Verbesserung des Gesamtüberlebens \\ bei allen histologischen Subtypen erreicht worden}

Immuncheckpoint-Kombinationen im Stadium IV

Bei Nichtvorliegen einer Treibermutation kommt als Systemtherapie sowohl beim NSCLC als auch beim kleinzelligen Lungenkarzinom (small-cell lung carcinoma, SCLC) eine sogenannte Immuncheckpoint-Che-
motherapie-Kombination in Betracht. Damit ist eine Verbesserung des Gesamtüberlebens bei allen histologischen Subtypen erreicht worden. Beim NSCLC stehen je nach Histologie mehrere ChemotherapieRegimes zur Verfügung (z.B. Cisplatin-haltig oder Carboplatin-haltig, verschiedene Taxane und teils VEGF-Inhibition). Beim NSCLC ist - unabhängig von der Histologie - bei hoher PD-L1-Expression mit >50\% eine alleinige Immuncheckpoint-Inhibition möglich; derzeit ist aber nur Pembrolizumab zugelassen. Ebenso wird zukünftig eine chemotherapiefreie Immuncheckpoint-Kombination aus PD-L1 und CTLA4-Inhibitor möglich sein (CheckMate227) [8]. Hervorzuheben sind hier erste Langzeit-Überlebensanalysen mit Immuntherapie von etwa 25\% über 5 Jahre [9].

Als realistisches Ziel für die nähere Zukunft kann die Senkung der Lungenkrebs-Gesamtmortalität formuliert werden. Ein Lungenkarzinom wird sich mehr und mehr zu einer chronischen Erkrankung entwickeln, mit der sehr viel mehr Patienten länger und besser (über)leben können als zuvor.

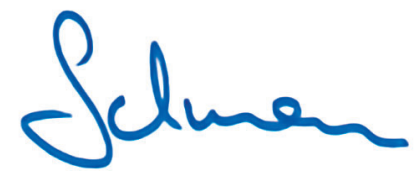

Prof. Dr. Christian Schumann

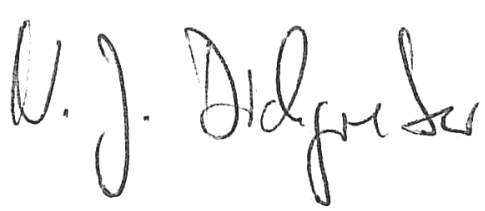

Dr. Nicolas J. Dickgreber

\section{Literatur}

$>1$ National Lung Screening Trial Research Team, Aberle DR, Adams AM, et al.: N Engl J Med. 2011 Aug 4;365(5):395-409.

2 Oudkerk M, Devaraj A, Vliegenthart R, et al.: Lancet Oncol 2017;18(12):e754-e766.

3 Antonia SJ, Villegas A, Daniel D, et al.: N Engl J Med 2018;379(24):2342-2350.

4 Gray JE, Okamoto I, Sriuranpong V, et al.: Clin Cancer Res 2019;25(22):6644-6652.

5 Peters S, Cho BC, Reinmuth N, et al.: AACR; Cancer Res 2019;79(13 Suppl):Abstract nr CT074

6 Frederickson AM, Arndorfer S, Zhang I, et al.: Immunotherapy 2019;11(5):407-428.
7 Paz-Ares L, Luft A, Vicente D, et al.: N Engl J Med 2018;379(21):2040-2051.

8 Hellmann MD, Ciuleanu TE, Pluzanski A. et al.: N Engl J Med 2018;378(22):2093-2104.

9 Garon EB, Hellmann MD, Rizvi NA, et al.: J Clin Oncol 2019;37(28):2518-2527. 